\title{
钯配合物催化的环丙烯活性/可控烯基加成聚合
}

\author{
侯小华吴宗铨* \\ (合肥工业大学化学与化工学院 先进催化材料与反应工程安徽重点实验室 合肥 230009)
}

\section{Living/Controlled Vinyl Addition Polymerization of Cyclopropenes Catalyzed by Palladium Complex}

\author{
Hou, Xiaohua Wu, Zongquan* \\ (Anhui Key Laboratory of Advanced Catalytic Materials and Reaction Engineering, School of Chemistry and \\ Chemical Engineering, Hefei University of Technology, Hefei 230009)
}

环丙烯是最小的不饱和碳氢环状化合物，由于其超 高的环张力 $(227.7 \mathrm{~kJ} / \mathrm{mol})$, 具有丰富的反应性 ${ }^{[1]}$, 在有 机合成和高分子合成中应用广泛, 尤其是其制备出的高 分子聚合物具有特殊的骨架结构. 但该方向的研究主要 聚焦于环丙烯单体发生开环易位聚合(ROMP)得到柔性 的不饱和碳链 ${ }^{[2]}$. 通过环丙烯烯基双键的加成聚合来制 备出饱和环状骨架结构的聚合物链则鲜有报道 ${ }^{[3]}$. 饱和 环状结构的刚性骨架能促进聚合物物理性能的提高, 例 如改善机械和热稳定性, 提高玻璃化转变温度, 以及获 得低的介电常数. 目前环烯烃的加聚反应几乎都局限于 降冰片烯和环戌烯结构, 而且只有少数的例子是活性聚 合过程. 因此实现环丙烯单体的活性/可控加成聚合仍 然是一个挑战性课题.

虽然早在 1970 年就已经有关于钯催化环丙烯类单 体的加聚反应的报道, 但只得到环状二聚体或者含有钯 的齐聚物 ${ }^{[4]} .1996$ 年, Risse 团队 ${ }^{[5]}$ 报道了二价阳离子钯 催化的 3,3-二烷基取代的环丙烯的加成聚合, 得到由三 元环重复单元组成的聚合物, 其拥有高分子量 $\left(M_{\mathrm{n}}=\right.$ 42000), 但其分子量分布很宽, 显示该聚合过程是不可 控的.

在钯催化环丙烯聚合中, 合成高分子量聚合物的最 大困难在于金属钯阳离子活性中心的稳定性和反应能 力, 它可能发生质子化或开环反应, 从而发生链转移或 链终止. Risse团队报道的钯配合物上的螯合配体对聚合 过程有重要影响. Xia 课题组 ${ }^{[2]}$ 也报道了在开环易位聚 合中，环丙烯上的取代基团能极大地影响聚合反应，尤 其是酯基上氧的鳌合作用可能影响链增长速率.
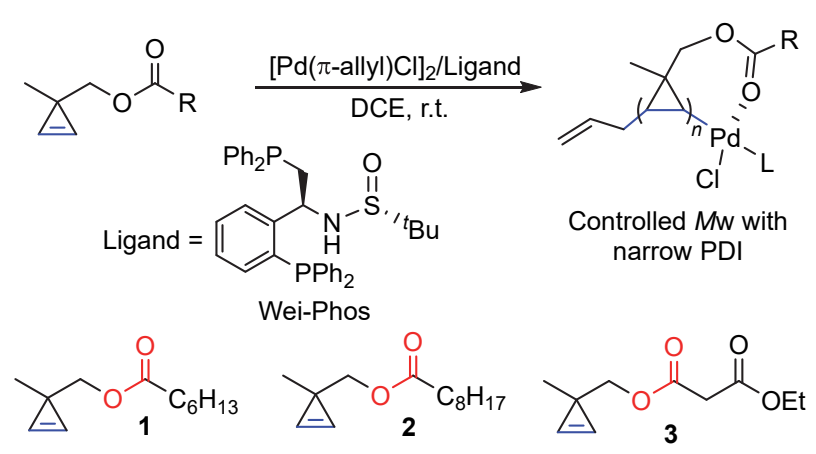<smiles>CC1(COC(=O)Cc2ccccc2)C=C1</smiles><smiles>[3H]C(C(=O)OCC1(C)C=C1)C(=O)OCC1(C)C=C1</smiles>

图式 1 钯催化体系实现环丙烯单体活性可控聚合 Scheme 1 Living/controlled vinyl addition polymerization by Pd

近日, 北京大学化学学院王剑波课题组 ${ }^{[6]}$ 在环丙烯 的烯基加成聚合领域取得了突破性的进展，该团队细致 调查了烯丙基氯化钯催化环丙烯单体的聚合过程, 发现 膦配体在其中起着至关重要的作用. 在无膦配体添加 下，相应聚合物分子量分布较宽，显示出其聚合可控性 较差, 而加入单膦配体 $\mathrm{P}^{t} \mathrm{Bu}_{3}$, 发现得到聚合物分子量 分布轻微变窄, 尤其是单膦配体的添加比例降低到与钯 配合物等物质的量时，获得聚合物的分子量接近理论 值，且分子量分布很窄，证明聚合过程趋向于可控. 聚 合体系中添加双膦配体(dppb, BINAP 等)的研究发现,

* Corresponding author. E-mail: zqwu@hfut.edu.cn. Published online November 30, 2021. 
聚合速率明显降低, 这一现象与双膦配体夹角和刚性有 关. 但随着膦配体的刚性和位阻增大, 所得聚合物分子 量分布更窄. 尤其是具有大位阻的手性双膦配体 $\left(\left(S, R_{S}\right)-\right.$ Wei-Phos)的使用 ${ }^{[7]}$, 聚合物分子量接近理论值, 且分布 仅为 1.13 , 这反映出所有的金属钯中心均能高效地引发 单体聚合(Scheme 1). 此催化体系在不同溶剂均能有效 地促使环丙烯单体发生聚合反应, 但相应聚合物的分子 量及分子量分布差异较大. 温度对分子量的影响很小, 只是低温反应需要更长的反应时间. 另外, 在没有惰性 气体的保护下, 反应仍能顺利发生, 说明活性中心具有 良好的稳定性. 不同结构的环丙烯单体 $(\mathbf{1} \sim \mathbf{8})$ 均能适用 于此催化体系, 高产率地得到聚合物, 且分子量分布在 1.14 1.39 范围内. 该类聚合物具有良好的溶解性和热 稳定性. 通过核磁谱图证明, 聚合物结构是环丙烯单元 发生了烯键加成.

值得注意的是，由单体 $\mathbf{1}$ 和单体 $\mathbf{4}$ 制得的聚合物数 均分子量随着单体和催化剂之间的投料比例的增加而 线性增长, 且聚合过程中分子量分布一直保持很窄, 这 有力证明该钯催化体系的聚合过程是可控的. 在动力学 实验中, 单体在 $9 \mathrm{~min}$ 内转化率达到 $90 \%$ 以上, 说明 $\operatorname{Pd}(\mathrm{II}) /\left(S, R_{S}\right)$-Wei-Phos 催化体系能高效催化环丙烯单体 发生活性可控的聚合过程. 另外, 通过顺序加料成功实 现制备嵌段共聚物. 这些结果都反映出该催化体系实现 环丙烯类化合物活性聚合的性质.

$$
\begin{aligned}
& \underbrace{\mathrm{R}^{1}} \frac{\mathrm{R}^{2}}{\mathrm{DCE}, \mathrm{N}_{2} \text {, r.t. }}
\end{aligned}
$$

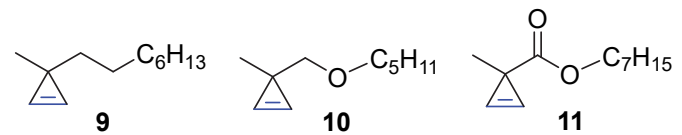

$$
\begin{aligned}
& \overbrace{12}^{\mathrm{Ph}} \stackrel{\mathrm{O}}{\mathrm{C}} \mathrm{C}_{6} \mathrm{H}_{13} \\
& \overbrace{13}^{\mathrm{O}} \overbrace{\mathrm{O}}^{\mathrm{O}} \overbrace{\mathrm{Ph}}
\end{aligned}
$$

图式 2 钯催化其它环丙烯单体的烯基加成聚合 Scheme 2 Pd-catalyzed vinyl addition polymerization of other cyclopropenes

对于单体、催化剂以及配体结构与聚合过程的内在 关系也予以系统研究. 单体取代基中的酯基与金属钯的 配位作用对实现可控聚合有着一定的作用，空间位阻过 大的取代基则阻碍配位作用，从而导致聚合物分子量分 布变宽, 也导致链引发和链增长速率过慢(Scheme 2). 催化体系中无需添加助催化剂 (AgOTf, NaBAr 4 , $\mathrm{AgSbF}_{6}$ ), 否则会致使聚合物分子量分布变宽, 反映出 聚合中氯离子仍在活性中心周围. 不同膦配体的聚合研
究反映其中的 $\mathrm{NH}$ 基团对聚合的可控性有一定的作用. 以此作者推断出合理的聚合过程(Scheme 3). 聚合中酯 基的弱配位作用对稳定活性中心、避免链转移和链终止 起到至关重要的作用 ${ }^{[8,9]}$.

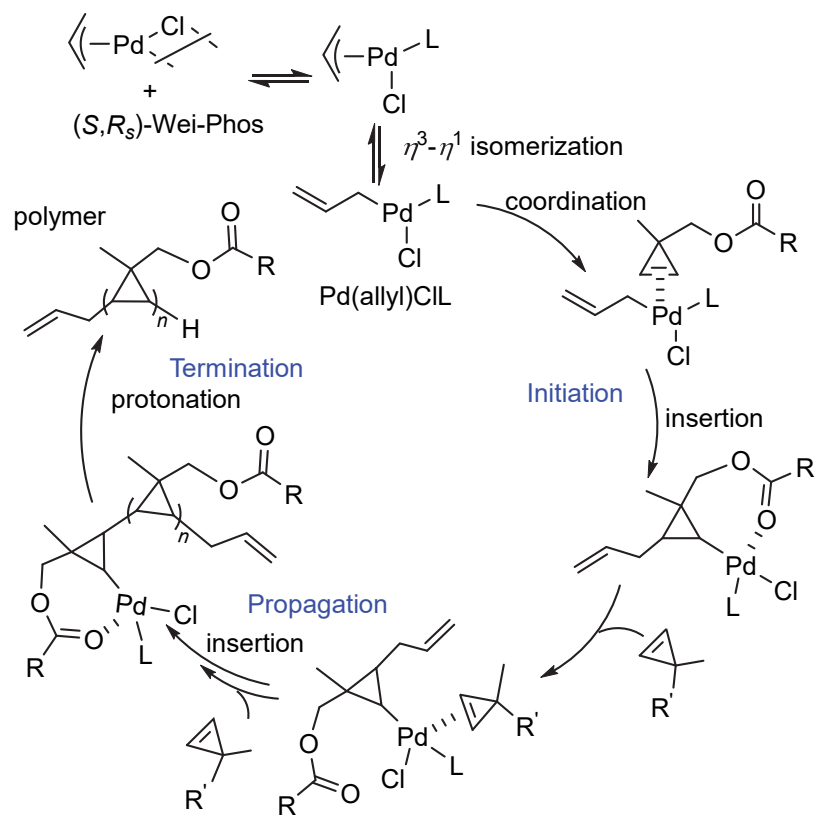

图式 3 聚合机理

Scheme 3 Mechanism for the polymerization

综上所述，王剑波课题组报道了含有双膦配体的 $[\mathrm{Pd}(\pi-\text { allyl }) \mathrm{Cl}]_{2}$ 催化体系能高效催化环丙烯单体发生活 性可控聚合, 得到的聚合物拥有饱和碳链和刚性的三元 环结构, 尤其是聚合物的数均分子量与单体的转化率呈 现线性关系，且聚合过程中分子量分布一直保持很窄. 另外，通过顺序加料成功实现制备环丙烯类单体的嵌段 共聚物. 研究表明膦配体与单体中羧基基团的协同配位 作用对实现高效活性可控聚合起着至关重要的作用.

\section{References}

[1] Baird, M. S. Chem. Rev. 2003, 103, 1271.

[2] Elling, B. R.; Su, J. K.; Xia, Y. Acc. Chem. Res. 2021, 54, 356

[3] Kaminsky, W.; Bark, A.; Däke, I. Stud. Surf. Sci. Catal. 1990, 56, 425.

[4] Baird, R. L.; Weigert, F. J.; Shapley, J. R. J. Am. Chem. Soc. 1970, 92, 6630 .

[5] Rush, S.; Reinmuth, A.; Risse, W.; O'Brien, J.; Ferro, D. R.; Tritto, I. J. Am. Chem. Soc. 1996, 118, 12230 .

[6] Zhang, Z.; Gao, Y.; Chen, S.; Wang, J. J. Am. Chem. Soc. 2021, $143,17806$.

[7] Zhou, W.; Su, X.; Tao, M.; Zhu, C.; Zhao, Q.; Zhang, J. Angew. Chem., Int. Ed. 2015, 54, 14853

[8] Chu, J.-H.; Xu, X.-H.; Kang, S.-M.; Liu, N.; Wu, Z.-Q. J. Am. Chem. Soc. 2018, 140, 17773.

[9] Li, N.-N.; Li, X.-L.; Xu, L.; Liu, N.; Wu, Z.-Q. Macromolecules 2019, 52, 7260 . 